\title{
Estimación del coste sanitario de las úlceras por presión en pacientes lesionados medulares*
}

\section{Estimation of the Sanitary Cost of Bedsores (Pressure Sores) in Patients with Medullary Lesions}

\section{Estimação do custo sanitário das úlceras de pressão em pacientes com lesão medular}

Fecha de recepción: 21-12-15 Fecha de aceptación: 07-02-16 Disponible en línea: 03-05-16 doi:10.11144/Javeriana.rgyps15-30.ecsu

Cómo citar este artículo:

Cabello Granado PA, Arévalo-Velasco JM. Estimación del coste sanitario de las úlceras por presión en pacientes lesionados medulares. Rev. Gerenc. Polít. Salud. 2016; 15(30): 60-67. http://dx.doi. org/10.11144/Javeriana.rgyps15-30.ecsu

\author{
Pablo Antonio Cabello-Granado** \\ José Manuel Arévalo-Velasco***
}

Artículo de reflexión que presenta los resultados de la investigación realizada, desde una perspectiva analítica, sobre la determinación del coste de tratamiento de las ulceras por presión según tipología para el financiador del tratamiento.

** Doctor en Ciencias Económicas y Empresariales por la Universidad de Castilla-La Mancha. Técnico F.A. Control de Gestión en el Área de Gestión Económica y Presupuestaria del Servicio de Salud de Castilla-La Mancha. Calle

60 Rio Guadiana, 4. Toledo 45007. España. Correo electrónico: pacabello@sescam.org España. 


\section{Resumen}

Objetivo: conocer el coste total de cada tipo de úlcera por presión, ya que es un tipo de herida crónica que se produce habitualmente en pacientes con poca movilidad. Método: se ha realizado una revisión retrospectiva de las historias clínicas del periodo 2008-2011 correspondiente al registro de actividad de cirugía plástica del Hospital de Parapléjicos. Resultados: la úlcera por presión isquiática es la más frecuentemente tratada $(43,27 \%)$ así como también la de menor coste total ( $€ 57$ 196,29). El coste más alto de una úlcera por presión por su localización corresponde a la sacra $(40,41 \%$, siendo la segunda en frecuencia, y el coste total es de $€ 112$ 012,96). Conclusiones: ante estos costes de tratamiento, se imponen campañas de prevención para intentar evitar este alto coste al Sistema Nacional de Salud.

Palabras clave: úlcera por presión; economía de la salud; costo de enfermedad; prevención terciaria; sistemas de salud; financiación de la atención de la salud

\section{Abstract}

Objective: getting to know the total cost of each type of bedsore (pressure sores), as it is a chronical injury commonly seen in patients with low mobility. Method: We realized a retrospective review of clinical records for the 2008 to 2011 period, corresponding to the plastic surgery activity records of the Paraplegic Hospital. Results: the ischial pressure sore is the most commonly treated $(43.27 \%)$, and also shows the lowest total cost ( $€ 57,196.29)$. The highest cost of a bedsore due to its location corresponds to sacral pressure sores $(40.41 \%$, being the second most frequent type, with a total cost of $€$ 112,012.96). Conclusions: Faced with these treatment costs, prevention campaigns are established in an attempt to prevent these high costs to the National Health System.

Keywords: pressure ulcer; health economics; cost of illness; tertiary prevention; health systems; healthcare financing

\section{Resumo}

Objetivo: conhecer o custo total de cada tipo de úlcera de pressão, ainda que são tipos de ferida crônica que ocorre habitualmente em pacientes com baixa mobilidade. Método: foi realizada uma revisão retrospectiva dos prontuários médicos no período 2008-2011 correspondente ao registro de atividades de cirurgia plástica do Hospital de Paraplégicos. Resultados: as úlceras por pressão isquiáticas são as mais frequentemente tratadas $(43,27 \%)$ bem como as de menor custo total ( $€ 57196,29)$. O custo mais alto de uma úlcera de pressão pela sua região corresponde à sacral $(40,41 \%$, sendo a segunda em frequência, e o custo total é de $€ 112$ 012,96). Conclusões: perante estes custos de tratamento, impõem-se campanhas de prevenção para tentar evitar este alto custo ao Sistema Nacional de Saúde.

Palavras-chave: úlceras de pressão; economia da saúde; custo de doenças; prevenção terciaria; sistemas de saúde; financiamento do atendimento em saúde 


\section{Introducción}

Actualmente las heridas se clasifican en: 1) traumáticas: laceraciones, avulsiones, 2) quemaduras: calor, químicas, eléctricas 3) crónicas: diabéticas, vasculares, por radiaciones ionizantes y por presión.

La úlcera por presión es un área de necrosis celular localizada, resultado de una alteración mecánica, vascular y linfática de la piel y de tejidos más profundos, situados entre el plano esquelético y una superficie externa resistente. El apoyo sobre esta provoca fuerzas de compresión y cizallamiento en las estructuras señaladas. Cuando la intensidad y el tiempo de aplicación de estas fuerzas supera cierto nivel crítico se produce la lesión tisular. Además, se reconocen factores locales y generales que modifican la resistencia de los tejidos a las citadas fuerzas, lo que hace de las úlceras por presión un fenómeno de etiología multifactorial (1-3).

Presiones permanentes sobre los tejidos pueden provocar lesiones, identificadas por su etiología como úlceras por presión. Su prevalencia en personas mayores residentes en instituciones ha sido estimada entre 2,3 y $28 \%$ en diferentes estudios (4). El primer estudio nacional de prevalencia de úlceras por presión en España (5) revelaba como resultados más destacados una prevalencia media de úlceras por presión en atención primaria de un $8,34 \%$ entre los pacientes que recibían atención domiciliaria, un 0,54\% en los mayores de 65 años y un $0,11 \%$ en los mayores de 14 años; a nivel hospitalario fue del $8,81 \%$ y a nivel sociosanitario de $7,6 \%$. Sin embargo, entre la población con problemas de movilidad estos valores son mayores; se ha estimado que de un 50 a un $80 \%$ de las personas con lesión medular desarrollarán una úlcera por presión (6-8). Estudios más recientes elevan estos porcentajes a valores que van de 62,4 a $85,7 \%$ (9), e igualmente, se indican rangos de recurrencia de úlceras por presión que oscilan entre 31 y $79 \%$ (10), cifras que por lógica son variables de unos centros a otros y entre distintos niveles asistenciales.

Por ello, incluso la menor de las estimaciones anteriores demuestra que las úlceras por presión son un problema de salud muy importante, especialmente entre los lesionados medulares que se ven obligados a prolongados periodos de sedestación. Resulta difícil establecer el porcentaje de úlceras causadas por una prolongada sedestación en una silla de ruedas, pero estimaciones surgidas en la bibliografía sugieren entre 36 y $50 \%$ para la población mayor en situación de riesgo (11). Con los datos que posee el Hospital Nacional de Parapléjicos, que es un hospital monográfico e integral para el manejo del paciente lesionado medular, y extrapolando los resultados a nivel nacional, se puede decir que en España la prevalencia de esta subpoblación se sitúa en torno a 1-2\% y una incidencia anual de uno por diez mil habitantes y año $(12,13)$.

El coste asociado al tratamiento de las úlceras por presión en Estados Unidos superó los 6,4 billones de dólares (14). Un estudio realizado en Reino Unido indicaba que el coste para el servicio nacional de salud se situaba entre 180 y 321 millones de libras esterlinas (entre 0,4 y $0,8 \%$ del gasto de sanidad) (15). En España existe un estudio de aproximación al coste del tratamiento de úlcera por presión según severidad (16), pero no existe ningún estudio de coste económico de esta patología en pacientes lesionados medulares. Por eso, queremos aportar con este estudio, realizado en el Hospital de Parapléjicos, el coste medio aproximado de una úlcera por presión según su localización, ya que puede ser fácilmente extrapolable al resto de unidades de lesionados medulares del país, y así poder estimar el porcentaje final del gasto sanitario total. 


\section{Material y método}

El tipo de estudio realizado es observacional, siendo la fuente de datos la revisión retrospectiva de las historias clínicas de todos los pacientes operados en el Servivio de Cirugía Plástica de úlcera por presión en el Hospital de Parapléjicos, en el periodo comprendido enero del 2008 y diciembre del 2011.

Los citerios de inclusión en el estudio han sido: pacientes lesionados medulares, mayores de dieciocho años, la severidad de la úlcera por presión en estadio grado IV, la dimensión de la úlcera por presión debe ser mayor de cinco centímetros, con indicación de intervención quirúrgica para el tratamiento. Los criterios de exclusión son la existencia de infección en la úlcera por presión, denegación de consentimiento informado para intervención quirúrgica, severidad de la úlcera por presión inferior al estadio grado IV, dismensión de la úlcera por presión inferior a cinco centímetros.

El coste se ha determinado sobre la base de datos estandarizados del año 2011. Se ha tenido en cuenta el coste por día de estancia hospitalaria, el coste de una cura convencional realizada a pie de cama del paciente y el coste de la intervención quirúrgica, tanto si es únicamente de limpieza como si es para realizar el tratamiento definitivo de la úlcera por presión: cierre de esta (tabla 1). Para la determinación de los costes se han conside- rado exclusivamente los costes directos de atención y tratamiento hospitalario en cada caso estudiado. Se han considerado los costes totales de cada proceso, además de tener en cuenta los gastos de material fungible y de personal requeridos para cada tratamiento. Los datos económicos necesarios mencionados anteriormente se han obtenido de la Unidad de Control de Gestión y del Servicio de Gestión Económica y Suministros del hospital. Los datos relativos a los costes del personal los ha facilitado el Servicio de Personal del hospital.

Por otro lado, una vez obtenidos los datos de coste medio de la úlcera por presión en lesionados medulares, estos podrían extrapolarse para poder llegar a estimar el coste global a nivel nacional, teniendo en cuenta los datos estadísticos sobre epidemiología de las úlceras por presión y la Encuesta sobre Discapacidades, Autonomía Personal y Situaciones de Dependencia que publica el Instituto Nacional de Estadística.

\section{Resultados}

En el estudio se han analizado las siguientes variables: se han contabilizado el número total de pacientes, edad, sexo, el número total de procedimientos quirúrgicos realizados con su media \pm desviación estándar y su moda, y finalmente la estancia hospitalaria

Tabla 1. Costes sanitarios del TRATAMIENTo de UNA ÚlCERA POR PREsión

\begin{tabular}{|l|c|}
\hline Costes sanitarios & $\mathbf{2 0 1 1}$ \\
\hline Estancia & $€ 525,63$ \\
\hline Cura & $€ 24,11$ \\
\hline IQ Limpieza $(*)$ & $€ 486,33$ \\
\hline IQ Cierre $(*)$ & $€ 964,46$ \\
\hline
\end{tabular}

IQ: Intervención quirúrgica

Fuente: elaboración propia a partir de los datos económicos de la Unidad de

Gestión Económica y Control de Gestión del Hospital de Parapléjicos 
Tabla 2. Características generales de la población de estudio

\begin{tabular}{|l|l|}
\hline Periodo de estudio & $2008-2011$ \\
\hline Número de pacientes & 245 \\
\hline Distribución por sexo & 205 V, 40 M (relación 5:1) \\
\hline Edad & $46 \pm 16$ años (rango 12-65) \\
\hline Total de cirugías & 609 \\
\hline Media \pm sD* de cirugías & $2,49 \pm 2,19$ \\
\hline Moda de cirugías & 1 \\
\hline Media \pm SD * de estancia Hospitalaria & $144,20 \pm 119,14$ \\
\hline
\end{tabular}

*SD: desviación estándar.

Fuente: elaboración propia a partir del registro de actividad interna de la Unidad de Cirugía Plástica del Hospital de Parapléjicos

con la media \pm desviación estándar y su moda (tabla 2).

Los principales resultados obtenidos se muestran en la tabla de datos referidos a cada tipo de úlcera por presión (tabla 3 ) y en la tabla de coste medio según el tipo de úlcera por presión (tabla 4), pero podemos señalar que la úlcera por presión más frecuentemente intervenida es la isquiática, la cual representa un 43,27\%. También en este caso el número medio de intervenciones realizadas es el más bajo: 2,17, y la estancia media hospitalaria es la más baja de todas, ya que ha sido de 97 días (tabla 3). Situándose en el lado contrario, con el menor porcentaje de tratamiento, se encuentran las denominadas "de otras loca- lizaciones" (sobre todo maleolos y occipital), que representan solo un 7,75\% (tabla 3). Cabe asimismo descatar que el tratamiento de la úlcera por presión que más estancia media hospitalaria consume es la úlcera por presión sacra (192 días) (tabla 3).

En lo que se refiere a coste medio por caso, las isquiáticas son las más baratas, ya que también lo es en sus curas, su estancia hospitalaria y tratamiento quirúrgico. Esto hace que tenga un coste medio total de $€ 57$ 196,29 (tabla 4), y en el extremo opuesto, la más cara es la sacra, $€ 112$ 012,96, seguida muy de cerca por la trocantérea, con $€ 105$ 992,48. Logicamente, esto es debido al elevado coste de sus curas, la elevada estancia hospitalaria

TABla 3. DAtos Referidos A CADA TIPO DE ÚlCERA POR PRESIÓN

\begin{tabular}{|l|c|c|c|c|c|c|}
\hline $\begin{array}{l}\text { Tipo de úlcera } \\
\text { por presión }\end{array}$ & $\begin{array}{c}\text { Número de } \\
\text { úlcera por } \\
\text { presión }\end{array}$ & $\begin{array}{c}\text { Número de in- } \\
\text { tervenciones }\end{array}$ & $\begin{array}{c}\text { Número de } \\
\text { estancias }\end{array}$ & $\begin{array}{c}\text { Porcentaje } \\
\text { tipo }\end{array}$ & $\begin{array}{c}\text { Número de in- } \\
\text { tervenciones } \\
\text { medio (*) }\end{array}$ & $\begin{array}{c}\text { Estancia } \\
\text { media }\end{array}$ \\
\hline Isquiática & 106 & 229 & 10269 & $43,27 \%$ & 2,17 & 97 \\
\hline Otras & 19 & 33 & 2272 & $7,75 \%$ & 1,74 & 120 \\
\hline Sacra & 99 & 276 & 19004 & $40,41 \%$ & 2,79 & 192 \\
\hline Trocantérea & 21 & 71 & 3784 & $8,57 \%$ & 3,39 & 181 \\
\hline Total & 245 & 609 & 35329 & $100,00 \%$ & 2,49 & 144,2 \\
\hline
\end{tabular}

(*) Una intervención quirúrgica de cierre, y el resto son intervenciones quirúrgicas medias de limpieza.

64 Fuente: elaboración propia a partir del registro de actividad interna de la Unidad de Cirugía Plástica del Hospital de Parapléjicos 
TABla 4. Coste Medio SEgún El TIPO de ÚlCERA POR PRESIÓN

\begin{tabular}{|l|c|c|c|c|}
\hline \multicolumn{5}{|c|}{ Coste por tipo úlcera por presión (€) } \\
\hline $\begin{array}{l}\text { Tipo úlcera por } \\
\text { presión }\end{array}$ & Cte. curas (**) & Cte. estancias & Cte. IQ (*) & Total CTE \\
\hline Isquiática & 4677 & 50986 & 1533 & 57196,29 \\
\hline Otras & 5786 & 63075 & 1324 & 70185,57 \\
\hline Sacra & 9257 & 100921 & 1835 & 112012,96 \\
\hline Trocantérea & 8727 & 95139 & 2127 & 105992,48 \\
\hline Total & 6953 & 75796 & 1689 & 84437,33 \\
\hline
\end{tabular}

(*) Una IQ de cierre, el resto de limpieza.

(**) Coste para dos curas diarias.

Fuente: elaboración propia a partir del registro de actividad interna de la Unidad de Cirugía Plástica y los datos económicos de la Unidad de Gestión Económica y Control de Gestión del Hospital de Parapléjicos

y también por el coste de las intervenciones quirúrgicas que precisan hasta alcanzar la curación (tabla 4).

\section{Discusión}

Actualmente en España no existe ningún estudio económico que evalúe el gasto sanitario de las úlceras por presión en pacientes lesionados medulares, tanto en su conjunto como de forma individualizada. El primer estudio nacional de prevalencia en España es el de Torra et al. (5), y este no define con certeza la prevalencia e incidencia de las úlcera por presión en concreto en la subpoblación de lesionados medulares. En este estudio, con los datos que disponemos de la actividad hospitalaria realizada tanto clínica como quirúrgica desde 1974, cuando fue abierto, hemos hecho un cálculo para extrapolar a nivel nacional la incidencia y prevalencia que manejamos en el Hospital de Parapléjicos, siendo estas de uno por mil y un $1-2 \%$ respectivamente (17).

En lo que respecta al análisis económico, este se ha realizado basado en valores referidos al año 2011 (tabla 1). Con estos datos hemos podido calcular de forma independiente el coste medio total según el tipo de localiza- ción de la úlcera por presión. Llamativamente, en este sentido hemos comprobado que la úlcera por presión más frecuente $(43,27 \%)$ es la isquiática, y es a la vez la que tiene un coste menor ( $€ 57196,29)$ (tablas 3 y 4). Estas son sin duda las más frecuentes entre varones de mediana edad que permanecen largos periodos de tiempo sentados, sin realizar pulsiones, y que además tienen habitualmente comorbilidad de enfermedades sistémicas como diabetes y/o enfermedades cardíacas que aumentan la mala vascularización periférica (tabla 2) (12). Les siguen en gasto sanitario las denominadas en otras localizaciones. Estas se refieren sobre todo a las occipitales y a los maleolos. Estas úlceras por presión habitualmente solo se desbridan y se deja que cicatricen por segunda intención. Son las más infrecuentes $(7,75 \%)$, pero tienen un coste más elevado que las isquiáticas (€ 70185,57) debido a que alargan su estancia hospitalaria al dejarlas evolucionar por segunda intención. En definitiva, más estancia y menos gasto en intervenciones quirúrgicas $(1,74)$ (tabla 3). En el otro extremo, las más caras son las úlceras por presión sacras y las trocantéreas. Las sacras porque se forman ya en los primeros días del ingreso en la UVI (40,41\% y 192 días de estancia). Son los pacientes agudos lesionados medulares de mayor estancia hospitalaria, porque no son 
dados de alta hasta que no se estabiliza su lesión y se les enseña a ser autónomos. Y la segunda por frecuencia y la de mayor coste (€ 112012,96) (tabla 4). Finalmente, las úlceras por presión trocantéreas, que son bastante infrecuentes $(8,57 \%)$, se producen en la fase crónica de la lesión medular. Como en el resto, sobre todo en varones de edad media cuya etiología habitual son los traumatismos al realizar las transferencias intradomiciliarias, al pasar al coche o por mantener el decúbito lateral de forma prolongada en la cama. Suelen ser muy grandes de tamaño, por lo que requieren la mayor cantidad de intervenciones quirúrgicas de todas $(3,39)$ (tabla 3). Y como es una zona móvil, ya que descansa el colgajo en la articulación coxofemoral, tarda tiempo en adherirse a esta, presentando frecuentemente serosas postoperatorias y dehiscencias, lo cual alarga la estancia hospitalaria hasta una media de 181 días, y esto supone un coste similar a la úlcera por presión sacra, que era la más cara (€ 105 992,48) (tabla 4) (18). Estas cifras de coste están muy por encima del estudio de aproximación al coste del tratamiento de úlcera por presión según severidad (16), donde se indica un coste medio de tratamiento en hospitalización para las lesiones de estadio IV de tan solo $€ 6802$. En nuestro caso, esta cifra es notablemente superior, ya que el estudio se centra en pacientes con lesión medular en estadio IV, en los que está indicado intervención quirúrgica, tanto de limpieza como de cierre, factor de coste muy importante que en un estudio de aproximación referenciado (16) no es tenido en cuenta, y que para este tipo de pacientes, lesionados medulares, es de vital importancia considerar. No obstante, a pesar del nivel de severidad existen, como hemos podido apreciar, diferencias importantes en el coste 66 del tratamiento de las úlceras por presión dependiendo de su localización.
Con estos datos a la vista, hemos realizado un cálculo del coste total medio anual, que hemos extrapolado a nivel nacional, estimando el gasto total nacional en úlcera por presión de lesionados medulares en torno a seiscientos millones de euros. Cifra muy similar, corrigiendo el desfase del tiempo y de la población, a la que aportan Bennet et al. (15). Sin embargo, la cifra de Marwick et $a l$. nos parece muy abultada, salvo si se piensa en la población de Estados Unidos en ese año y si se incluyen todo tipo de úlceras, aun heridas crónicas, y no solo pacientes lesionados medulares (14). Hortelano Otero et al. aportan la cifra de 461 millones de euros en un artículo que no es de carácter económico. Se desconoce el origen de esta cifra (19).

\section{Conclusiones}

Este artículo trata por primera vez del análisis de los costes sanitarios de las úlceras por presión individualizado por su localización. El 80\% de los pacientes tratados son varones, con una edad media de $46 \pm 16$ años. La úlcera más frecuente es la isquiática $(43,27 \%)$, producida habitualmente por un exceso de horas de sedestación. Por lo tanto, este grupo de pacientes son los más afectados y los que más demandan un tratamiento quirúrgico, con un coste medio total de $€ 57196,29$. En el otro extremo se sitúan las úlceras por presión trocantéreas $(8,57 \%)$, producidas sobre todo por traumatismos en esa localización al realizar transferencias, con un coste medio total de $€ 105992,48$, que representa casi el doble de las isquiáticas, debido a la elevada estancia hospitalaria así como por el número de intervenciones quirúrgicas que precisa. Estos aspectos nos han llevado a calcular un coste total anual para todas las úlceras por presión tratadas en España en torno a los seiscientos millones de euros, en lo que se refiere únicamente a la subpoblación de lesionados medulares, lo que puede repre- 
sentar en torno a un $1 \%$ del gasto sanitario total en España. Estos hallazgos nos llevan a promover la utilización de campañas de prevención que insistan frecuentemente en qué es una úlcera por presión, por qué se produce y cuál es su coste y su tratamiento quirúrgico, ya que pueden ser tan baratas como la décima parte del coste total de una úlcera por presión y alcanzar a una población diana de cincuenta a cien lesionados medulares por campaña.

\section{Referencias bibliográficas}

1. Bea M, Salvador MP, Pascual V. Prevención y tratamiento de las úlceras por presión en el paciente inmovilizado. Rehabilitación. 1995; 29 (6).

2. Brienza D, Geyer M, Karg P. White paper on pressure management. En Wheelchair seating: A state of the science conference on seating issues for persons with disabilities . Pittsburgh, PA: University of Pittsburgh; 2001.

3. Cullum N. Support surfaces for pressure ulcer prevention (review). The Cochrane Collaboration. Wiley Publishers; 2005.

4. Young JS, Burns PE. Pressure sores and the spinal cord injured. sCI Digest. 1981; 3: 11-23.

5. Torra JE, Rueda J, Soldevilla JJ, Martínez Cuervo F, Verdú J. Primer Estudio Nacional de Prevalencia de úlceras por presión en España. Epidemiología y variables definitorias de las lesiones y pacientes. Gerokomos. 2003; 14 (1): 37-47

6. Richardson RR, Meyer PR Jr. Prevalence and incidence of pressure sores in acute spinal cord injuries. Paraplegia. 1981 Mar; 32 (3): 150-8.
7. Rodríguez GP, Garber SL. Prospective study of pressure ulcer risk in spinal cord injury patients. Paraplegia. 1994 Mar; 32 (3): 150-8.

8. Salzberg CA, Byrne DW, Cayten CG, et al. A new pressure ulcer risk assessment scale for indiciduals with spinal cord injury. Am J Phys Med Rehabil. 1996; 75 (2): 96-104.

9. Rappl L. Physiological changes in tissues denervated by spinal cord inyury tissues and possible effects on wound healing. Int Wound J. 2008; 5 (3): 435-44.

10. Bates-Jensen B, Guihan M, Garber SL, Chin A, Burns S. Characteristics of recurrent pressure ulcers in veterans with spinal cord injury. Spinal Cord Med. 2009; 32 (1): 34-42.

11. Zacharkow D. Wheelchair posture and pressure sores. Springfield, IL: Charles C. Thomas; 1984.

12. Arévalo JM, Espino FJ. Estudio clínico retrospectivo del uso de un sistema de hidrocirugía en pacientes lesionados medulares con úlceras por presión crónicas. Cir Plast Iberolatinoam 2011; 37 (1): 1-6.

13. Arévalo JM, Espino FJ. Manejo multidisciplinario de las úlceras por presión en pacientes medulares. Heridas y Cicatrización. 2011; 2 (5): 15-9.

14. Marwick C. Recomendations for pressure sores. JAMA. 1992; 268: 700-1.

15. Bennet G, Dealey C, Posnett J. The cost of pressure ulcers in the UK. Age and Ageing. 2004; 33: 230-5.

16. Soldevilla J, et al. Una aproximación al impacto del coste económico del tratamiento de las úlceras por presión en España. Gerokomos. 2007; 18 (4): 43-52.

17. Langemo DK, Melland H, Hanson D, Olseon B, Hunter S. The lived experience of having a pressure ulcer: A quantitative analysis. Advances in Skin and Wound Care. 2000; 13: 225-35.

18. Saunders L, Krause J, Peters B, Reed K. The relationship of pressure ulcers, race and socioeconomic conditions after spinal cord injury. J Spinal Cord Med. 2010; 33 (4): 387-95.

19. Hortelano Otero A, Centeno Silva JA, Lorca García C, et al. Pautas para el uso de terapia de vacío en el Servicio de Cirugía Plástica del Hospital La Fe en Valencia (España). Cir Plast Iberolatinoam 2010; 36 (2): 97-106. 